\title{
Avaliação de hipsômetros e operadores na mensuração de árvores de Eucalyptus
} urograndis de tamanhos diferentes

\section{Evaluation of hypsometers and operators in measuring tree heights in Eucalyptus urograndis with different sizes}

\author{
Rafaella Carvalho Mayrinck ${ }^{1 *}$, Ximena Mendes de Oliveira ${ }^{2}$, Gabriela Cristina Costa Silva ${ }^{3}$, Paula Carolina Gomides Vitor ${ }^{4}$, \\ Antonio Carlos Ferraz Filho
}

Resumo: A mensuração da altura de forma acurada é extremamente importante na ciência florestal, pois, influencia diretamente na predição do volume individual das árvores, bem como na volumetria de um povoamento e em todo o sistema de prognose. Por ser feita de forma indireta, é sujeita a erros, que devem ser minimizados. Assim, deve se usar o hipsômetro que proporcione menor erro, além de um operador treinado para o procedimento. Nesse estudo objetivou-se testar a acurácia do hipsômetro e operador em relação a árvores de Eucalyptus urograndis de tamanhos diferentes. Os dados foram coletados em um plantio de Eucalyptus urograndis em Turmalina, MG. Três árvores foram selecionadas, classificadas em pequena (12,1 m), média $(18,9 \mathrm{~m})$ e grande $(25,2 \mathrm{~m})$. Seis operadores mediram cada uma das árvores usando 4 hipsômetros (Prancheta dendrométrica, Clinômetro digital, Suunto e Vertex) 5 vezes. Posteriormente, realizou-se o abate das árvores e a obtenção da altura real com uma trena. Para analisar os dados, foi feita uma análise de variância, teste de Tukey e teste T. Os resultados mostram que houveram diferenças entre os valores encontrados pelos observadores, o que salienta a necessidade de treinamento. Todos os instrumentos geraram valores confiáveis. Para árvores mais baixas, o Vertex foi o instrumento mais confiável e para árvores maiores o Clinômetro digital foi o instrumento mais indicado.

Palavras-chave: Hipsômetro; Instrumentos dendrométricos; Mensuração florestal; Biometria.

\begin{abstract}
The accurate mensuration of tree hight is very important in forest science because it influences directly the individual tree volume, stand volume prediction and all the prognosis system. Due to the fact it is made indirectly, errors can occur, and they should be minimized. Hence, one should use hypsometers, which lead to a minor error and a trained operator to do it. This study aims to test the accuracy of hypsometers and operator, regarding the size of the tree, classified as small (12,1 $\mathrm{m})$, medium $(18,9 \mathrm{~m})$ and large $(25,2 \mathrm{~m})$. Data was of 3 trees from a Eucalypus urograndis plantation in Turmalina, MG. Six operators measured the trees using 4 hypsometers (Dendrometric clipboard, Digital clinometer, Suunto and Vertex) 5 times each. The trees were felled and the real height of the trees were taken using a measure tape. Data was analysed by Analysis of variance, Tukey test and $\mathrm{T}$ test. The results showed difference between values found by different operators and highlight the need for training. All hypsometers were accurate. Measuring smaller trees, Vertex was the best one and measuring the higher ones, Digital clinometer was the best.
\end{abstract}

Key words: Hypsometer; Dendrometric instruments; Forest measurement; Biometric.

\footnotetext{
*Autor para correspondência

Recebido para publicação em 26/01/2016; aprovado em 20/12/2016

${ }^{1 * E s t u d a n t e ~ d e ~ M e s t r a d o ~ e m ~ E n g e n h a r i a ~ F l o r e s t a l, ~ U n i v e r s i d a d e ~ F e d e r a l ~ d e ~ L a v r a s, ~} 35$ 992175944, rafamka@ hotmail.com

${ }^{2}$ Estudante de Mestrado em Engenharia Florestal, Universidade Federal de Lavras, ximena_mendes@hotmail.com

${ }^{3}$ Estudante de Mestrado em Engenharia Florestal, Universidade Federal de Lavras, gbcristina.silva@gmail.com

${ }^{4}$ Estudante de Doutorado em Engenharia Florestal, Universidade Federal de Lavras, paulagvitor@ hotmail.com

${ }^{5}$ Professor, Universidade Federal de Lavras, antoniocarlos.ferraz@dcf.ufla.br
} 


\section{INTRODUÇÃO}

O inventário florestal é fonte de dados para o planejamento florestal. É por meio dele que se obtêm informações relacionadas à saúde, produtividade e produção de uma floresta. Entretanto, como qualquer procedimento na cadeia produtiva, é dotado de falhas que são objeto de investigação em diversos estudos.

Altura total e diâmetro à altura do peito (DAP) são as principais variáveis dendrométricas mensuradas no inventário florestal e utilizadas na quantificação do estoque presente e futuro de um povoamento (SANQUETTA et al., 2014). Assim, é extremamente importante que estas sejam mensuradas de forma a minimizar os erros.

A mensuração da altura total de árvores é tema de diversos estudos onde busca-se por aparelhos que retornem valores mais acurados (COUTO; BASTOS, 1998; JESUS et al., 2012; SILVA et al., 2012a; OLIVEIRA et al., 2014). É tema pertinente na ciência florestal, já que erros de 1 metro na mensuração da altura de árvores resultam em erros de aproximadamente $14 \%$ no volume da arvore (COUTO; BASTOS, 1998).

A mensuração da altura, feita de forma indireta, é um dos principais problemas que interferem na acuracidade do inventário (JESUS et al., 2012; OLIVEIRA et al., 2014). Diversos instrumentos de medição vêm sendo estudados há décadas (COUTO; BASTOS, 1998; JESUS et al. 2012; ANDRADE et al. 2016; FELICIANO et al., 2016) e ainda hoje busca se por um aparelho que seja acurado e eficiente em todos os casos, embora os instrumentos tenham se modernizado e aperfeiçoado com o passar do tempo.

Os instrumentos utilizados para obtenção da altura são os hipsômetros, que podem seguir princípios trigonométricos e geométricos. Os hipsômetros baseados em princípios geométricos utilizam para medição de altura, relações existentes entre triângulos semelhantes e os baseados em princípios trigonométricos consideram a determinação de ângulos formados entre a base, o topo da árvore e o nível do instrumento (MACHADO; FIGUEIREDO FILHO, 2003). Os instrumentos Haga, Suunto, Clinômetro, BlumeLeiss e Vertex estão entre os hipsômetros que seguem o princípio trigonométrico (BRACK, 2008).

Os erros mais comuns na obtenção da altura são ocasionados pela dificuldade em visualizar a base e o topo da árvore, presença de árvores inclinadas e mistura de copas em povoamentos mais adensados (SCOLFORO; THIERSCH, 2004). Para diminuir os erros é importante que os operadores sejam submetidos a treinamento com os aparelhos e também que estes sejam escolhidos de forma a reduzir os erros de medição.

Assim objetivou-se verificar a acurácia de quatro hipsômetros na medição de alturas de árvores de tamanhos diferentes (pequena, média e grande), verificando a influência do operador na mensuração da altura das árvores.

\section{MATERIAL E MÉTODOS}

\section{Caracterização da área e amostragem}

Os dados foram coletados de um povoamento de 388,94 hectares Eucalyptus urograndis, com espaçamento de 3 x 3 m e 9 anos de idade, em Turmalina, MG, Brasil. Turmalina é classificada como Aw segundo a Köppen e Geiger, com temperatura média de $21.9^{\circ} \mathrm{C}$ e média anual de pluviosidade de $955 \mathrm{~mm}$ (CLIMATE DATA, 2016).

As alturas totais de três árvores, uma de tamanho pequeno (12,1 metros, árvore 1), uma de tamanho médio (18,9 metros, árvore 2) e uma árvore de tamanho grande $(25,2$ metros, árvore 3) foram mensuradas por 6 operadores (a,b,c,d,e,f), sendo que cada um usou 4 instrumentos diferentes (Prancheta dendrométrica, Clinômetro digital, Suunto e Vertex) na mensuração de cada uma das três árvores. A distância de mensuração entre a árvore e o operador foi de aproximadamente o tamanho da árvore, inferida pelo operador no campo, antes da medição. Cada árvore foi mensurada 5 vezes por cada operador, utilizando cada um dos instrumentos listados. Após esta etapa, as árvores foram abatidas e a altura total real foi obtida usando a trena.

\section{Avaliação estatística}

Os dados foram processados no software $\mathrm{R}$ ( $\mathrm{R}$ Core Team, 2016). A análise de variância foi realizada em esquema fatorial em blocos (operador considerado como bloco), a fim de se verificar se houveram diferenças significativas entre os valores obtidos pelos diferentes operadores com cada um dos instrumentos utilizados e a interação entre estes. Posteriormente, foi realizado o teste de Tukey a $5 \%$ de probabilidade para verificar diferenças entre os instrumentos. Para verificar se houveram diferenças significativas entre alturas estimadas por cada instrumento e o valor real das alturas (mensuradas com trena após abate), foram feitos testes t de Student, a $95 \%$ de confiança.

\section{RESULTADOS E DISCUSSÃO}

Através da análise de variância (Tabela 1) foram verificadas diferenças significativas entre os instrumentos, operadores e obviamente entre os valores de altura das árvores mensuradas. As diferenças entre os operadores evidenciam a importância de um treinamento adequado no uso de cada aparelho para reduzir o erro na mensuração. Não houve diferença significativa na interação operador $x$ instrumento.

Tabela 1. Análise de variância dos dados de alturas obtidas por diferentes hipsômetros na medição de três árvores de Eucalyptus urograndis de tamanhos diferentes, em Turmalina, MG, Brasil.

\begin{tabular}{cccccc}
\hline Fonte de variação & GL & SQ & QM & F calc & F crítico \\
\hline Árvore & 4 & 98 & 25 & 19.27 & $2.56 \mathrm{e}-14 * * *$ \\
Bloco (operador) & 2 & 9041 & 4521 & 3543.94 & $2 \mathrm{e}-16 * * *$ \\
Hipsômetros & 3 & 106 & 35 & 27.64 & $4.91 \mathrm{e}-16 * * *$ \\
Operador x Hipsômetro & 6 & 1 & 0 & 0.18 & 0.982 \\
Resíduo & 344 & 439 & 1 & & \\
\hline
\end{tabular}

Onde GL: graus de liberdade; SQ: soma de quadrados; QM: quadrado médio; F calc: Valor de F calculado; F critico: valor de F crítico. 
A Tabela 2 mostra o teste Tukey feito a fim de comparar valores obtidos pelos instrumentos e apurar se existem diferenças significativas entre eles. De acordo com a Tabela 2, valores obtidos pelo Vertex não diferem estatisticamente de valores obtidos pela prancheta dendrométrica. Valores obtidos pelo Suunto e valores obtidos pelo Clinômetro digital diferem entre si e entre os valores obtidos pelos instrumentos anteriores. Valores obtidos pelo Suunto são estatisticamente maiores que valores obtidos pelo Clinômetro digital, e estes são estatisticamente maiores que valores obtidos pelo Vertex e pela Prancheta dendrométrica. A altura média das árvores usadas no experimento foi de 18,7 metros. Assim, o aparelho que obteve valor mais próximo da média das alturas usadas no experimento foi o aparelho Suunto. Feliciano et al. (2016) ao comparar medições por meio de instrumentos com a altura real de três varas telescópicas com 4,45; 7,8 e 10,75 metros verificaram que o Suunto foi estatisticamente semelhante ao comprimento real em todos os casos.

Tabela 2. Teste de Tukey para comparar as médias obtidas por diferentes hipsômetros na medição de alturas de três árvores de Eucalyptus urograndis de tamanhos diferentes, em Turmalina, MG, Brasil.

\begin{tabular}{ccc}
\hline Instrumento & Média & Resultado do teste \\
\hline Suunto & 18,85 & $\mathrm{a}$ \\
Clinômetro dendrométrico & 18,28 & $\mathrm{~b}$ \\
Vertex & 17,77 & $\mathrm{c}$ \\
Prancheta dendrometrica & 17,41 & $\mathrm{c}$ \\
\hline
\end{tabular}

Foi realizado um teste $\mathrm{t}$ a fim de se verificar as diferenças entre as alturas mensuradas indiretamente pelos aparelhos e as alturas obtidas pela mensuração direta com trena, após o abate das árvores (Tabela 3). Os valores maiores que o valor de $\mathrm{t}$ tabelado a $95 \%$ de confiança $(\mathrm{n}=30,2,776)$ indicam que a altura mensurada pelo instrumento foi estatisticamente diferente da altura real obtida por trena (valores em negrito na Tabela 3 ).
Conforme Tabela 3, o Vertex foi o aparelho que apresentou valores mais próximos ao valor real obtido por trena, para a árvore 1. A medida que a altura das árvores aumentou (árvores 2 e 3) todos os instrumentos tenderam aumentar o erro. Da mesma forma, Silva et al. (2012b), avaliando a influência de diferentes fontes de variação sobre as medições de alturas de árvores de Eucalyptus urophila $\mathrm{x}$ Eucalyptus grandis observaram que o erro foi maior para as classes de árvores mais altas.

Tabela 3. Resultado do teste t para comparar as médias obtidas por diferentes hipsômetros na medição de alturas de três árvores de Eucalyptus urograndis de tamanhos diferentes, em Turmalina, MG, Brasil. (Valores em negrito indicam diferença significativa entre a altura real e o valor obtido pelo instrumento).

\begin{tabular}{|c|c|c|c|c|c|}
\hline \multirow{2}{*}{ Árvore } & \multirow{2}{*}{ Operador } & \multicolumn{4}{|c|}{ Instrumentos } \\
\hline & & Prancheta & Clinômetro & Suunto & Vertex \\
\hline \multirow{6}{*}{1} & $\mathrm{a}$ & $-3,227$ & 1,002 & 0,467 & 0,382 \\
\hline & $\mathrm{b}$ & 0,988 & 3,264 & 0,651 & $-0,334$ \\
\hline & $\mathrm{c}$ & $-1,828$ & $-0,064$ & 2,798 & $-2,874$ \\
\hline & d & $-3,570$ & 1,055 & 0,733 & $-0,585$ \\
\hline & $\mathrm{e}$ & $-0,573$ & $-4,579$ & $-1,217$ & $-2,058$ \\
\hline & $\mathrm{f}$ & $-1,000$ & $-0,803$ & 4,745 & $-2,526$ \\
\hline \multirow{6}{*}{2} & $\mathrm{a}$ & $-5,189$ & $-0,732$ & $-4,782$ & $-2,072$ \\
\hline & $\mathrm{b}$ & $-1,235$ & 1,224 & $-0,623$ & $-4,090$ \\
\hline & $\mathrm{c}$ & $-3,113$ & $-2,948$ & 1,429 & $-6,915$ \\
\hline & $\mathrm{d}$ & $-5,317$ & $-2,337$ & $-0,489$ & $-1,902$ \\
\hline & $\mathrm{e}$ & $-2,417$ & $-8,072$ & $-2,781$ & $-7,203$ \\
\hline & $\mathrm{f}$ & $-3,250$ & $-4,418$ & 2,804 & $-5,298$ \\
\hline \multirow{6}{*}{3} & $\mathrm{a}$ & $-4,894$ & $-0,342$ & $-5,374$ & $-2,072$ \\
\hline & $\mathrm{b}$ & $-1,235$ & 1,224 & $-0,748$ & $-4,090$ \\
\hline & $\mathrm{c}$ & $-3,113$ & $-2,948$ & 1,429 & $-6,915$ \\
\hline & d & $-5,317$ & $-2,337$ & $-0,489$ & $-1,902$ \\
\hline & $\mathrm{e}$ & $-2,417$ & $-8,420$ & $-2,781$ & $-7,203$ \\
\hline & $\mathrm{f}$ & 2,186 & $-4,418$ & 2,804 & $-4,156$ \\
\hline
\end{tabular}

A Figura 1 mostra os valores de altura da menor árvore obtidos por cada um dos instrumentos utilizados e por cada operador (a, b, c, d, e, f) e o valor de altura real. Percebe-se que na maioria dos casos, a Prancheta dendrométrica e o Vertex subestimaram a altura real da árvore. O Clinômetro digital estimou valores que variaram em torno da média de forma aproximadamente homogênea e o Suunto superestimou valores de altura em comparação com a altura real, na maioria dos casos. Oliveira et al., (2014) estudaram diferentes aparelhos hipsométricos na medição da altura de árvores e concluíram que a Prancheta dendrometrica e o Clinômetro digital subestimaram as alturas das árvores. 
Figura 1. Valores de alturas da árvore 1 obtidos por cada operador utilizando os quatro instrumentos de medição e valor real obtido com trena, em um povoamento de Eucaliptus urograndis, em Turmalina, MG.

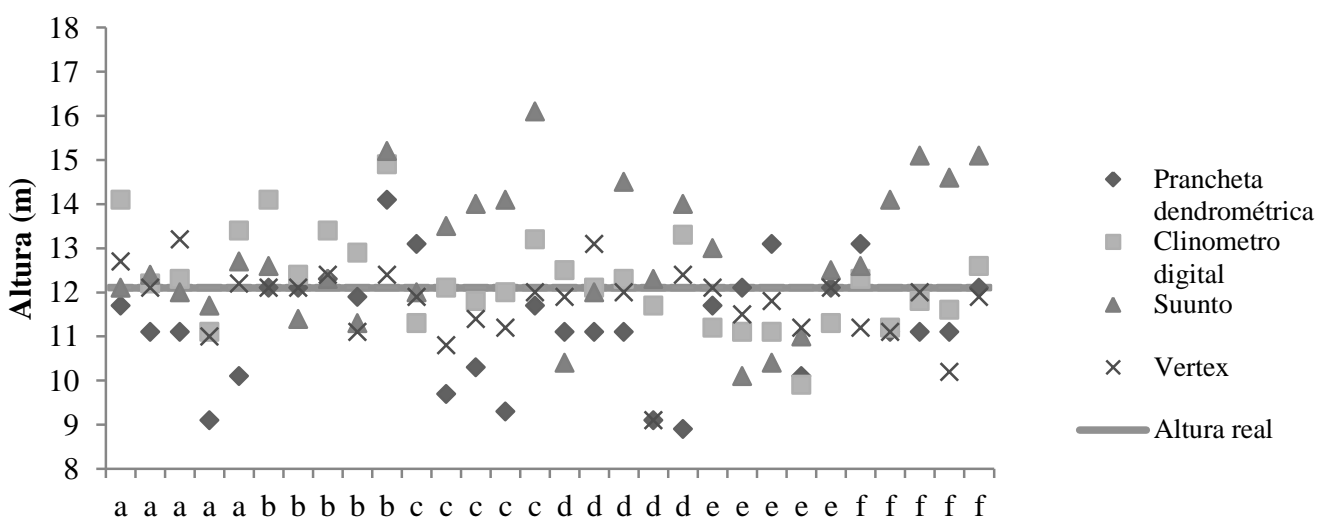

Operador

A Figura 2 mostra os valores de altura da árvore 2 e a Figura 3 mostra valores para a árvore 3, obtidos por meio dos diferentes instrumentos utilizados para cada operador (a, b, c, d, e, f). Nota-se que em ambos os casos, a Prancheta dendrométrica, o Vertex e o Clinômetro digital resultaram em subestimativas do valor real de altura da árvore. O Suunto estimou valores que variaram em torno da média de forma aproximadamente homogênea. De forma similar, Jesus et al. (2012) estudaram três aparelhos hipsométricos (Haga, Suunto e Vertex) em um povoamento clonal de híbrido Eucalyptus urophylla $\mathrm{x}$ Eucalyptus grandis no Distrito Federal/BR e verificaram que o Sunto obteve medidas mais próximas dos valores reais.

Figura 2. Valores de alturas da árvore 2 obtidos por cada operador utilizando os quatro instrumentos de medição e valor real obtido com trena, em um povoamento de Eucaliptus urograndis, em Turmalina, MG.
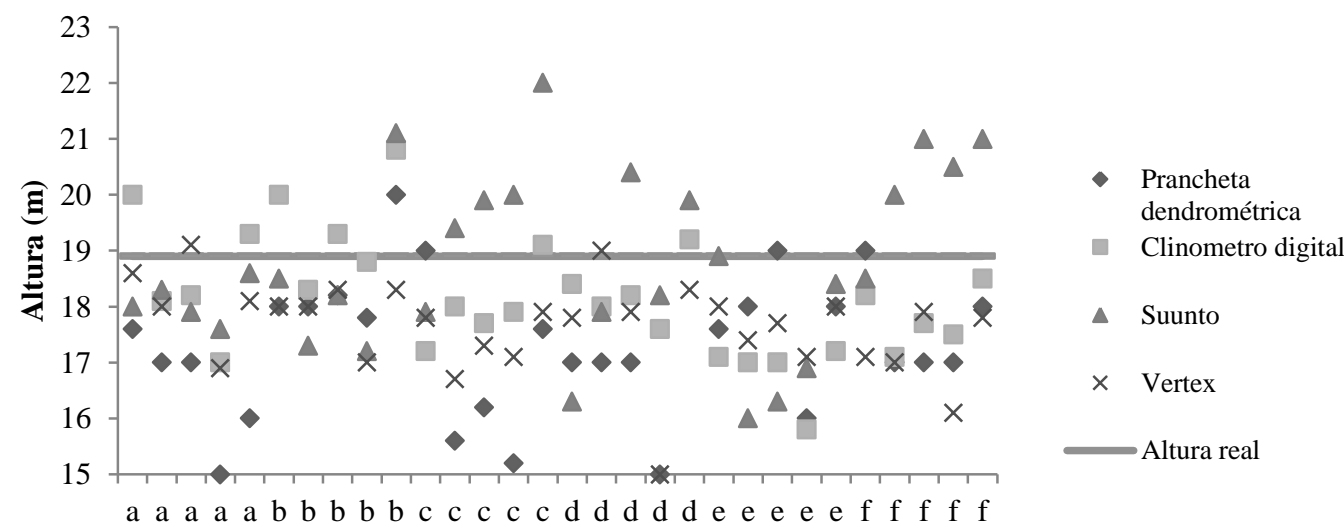

Operador

Figura 3. Valores de alturas da árvore 3 obtidos por cada operador utilizando os quatro instrumentos de medição e valor real obtido com trena, em um povoamento de Eucaliptus urograndis, em Turmalina, MG.

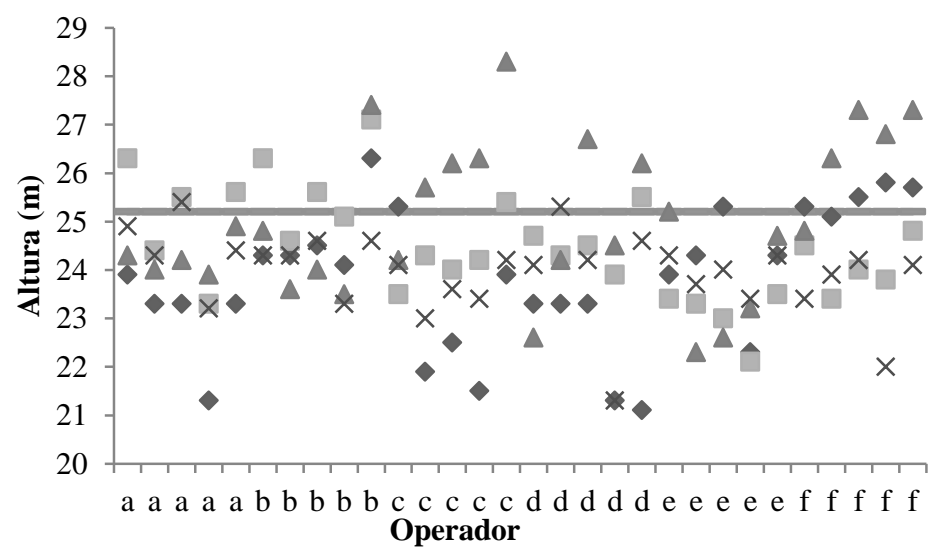

- Prancheta dendrométrica

- Clinometro digital

$\triangle$ Suunto

$\times \quad$ Vertex

Altura real

Operador 
A Tabela 4 apresenta a soma do quadrado do erro (SQE), o quadrado médio do erro (QME) e o erro padrão residual (Syx) em metros e em porcentagem. As estatísticas mostram que para a menor árvore, o Vertex foi o instrumento que resultou em menor erro. Para as árvores média e grande, o clinômetro foi o aparelho que apresentou o menor erro.
Todos os instrumentos foram confiáveis na mensuração da altura total da árvore e foram dependentes dos operadores, o que reforça a necessidade de um treinamento adequado dos operadores, como também foi visto no estudo de Oliveira et al. (2014).

Tabela 4 - Soma do quadrado do erro, quadrado médio do erro, erro padrão residual (m) e erro padrão residual (\%) de alturas obtidas por diferentes hipsômetros na medição de alturas de três árvores de Eucalyptus urograndis de tamanhos diferentes, em Turmalina, MG, Brasil.

\begin{tabular}{|c|c|c|c|c|c|}
\hline \multirow{2}{*}{ Árvore } & \multirow{2}{*}{ Estatística } & \multicolumn{4}{|c|}{ Instrumento } \\
\hline & & Prancheta & Clinômetro & Suunto & Vertex \\
\hline \multirow{4}{*}{1} & SQE & 68,64 & 34,48 & 87,11 & 23,88 \\
\hline & QME & 2,37 & 1,19 & 3,00 & 0,82 \\
\hline & Syx (m) & 1,54 & 1,09 & 1,73 & 0,91 \\
\hline & Syx $\%$ & 12,71 & 9,01 & 14,32 & 7,50 \\
\hline \multirow{4}{*}{2} & SQE & 136,86 & 51,22 & 71,63 & 67,62 \\
\hline & QME & 4,72 & 1,77 & 2,47 & 2,33 \\
\hline & Syx (m) & 2,17 & 1,33 & 1,57 & 1,53 \\
\hline & Syx \% & 11,49 & 7,03 & 8,32 & 8,08 \\
\hline \multirow{4}{*}{3} & SQE & 121,13 & 52,05 & 73,66 & 68,1 \\
\hline & QME & 4,18 & 1,79 & 2,54 & 2,35 \\
\hline & Syx (m) & 2,04 & 1,34 & 1,59 & 1,53 \\
\hline & Syx \% & 8,11 & 5,32 & 6,32 & 6,08 \\
\hline
\end{tabular}

\section{CONCLUSÃO}

Os instrumentos utilizados geraram valores confiáveis. Para árvores mais baixas, o Vertex foi $\mathrm{o}$ instrumento mais confiável e para árvores maiores o Clinômetro digital foi o instrumento mais indicado. No geral, o Suunto é o instrumento que obteve médias mais parecidas com as médias dos valores observados. É importante o treinamento dos operadores, devido às diferenças nos valores obtidos para a altura das árvores.

\section{REFERÊNCIAS}

ANDRADE, V. C. L.; RIBERO, J. R.; PINTO, I DE O.; SANTOS, M. J. F.; TELLES, L. B.; TERRA, D. L. C. V. Hipsômetros baseados no princípio geométrico avaliados em área de cerrado sensu stricto. Nativa, Sinop, v. 4, n. 5, p. 333336, 2016.

BRACK, C. Comparing diameter measuring instruments. Disponível em: <http://online.anu.edu.au/Forestry/mensuration/toolsd.htm>. Acesso: 7 jan. 2016.

CLIMATE DATA.ORG. Clima: Turmalina. Disponível em: https://pt.climate-data.org/location/24995/. Acesso: 31 jan. 2017.

COUTO, H. T. Z.; BASTOS, N., L., M. Erros de medição de altura em povoamentos de Eucalyptus em região plana. IPEF, n.39, p.21-31, ago.1998.

FELICIANO, M. E.; RIBEIRO, A.; FERRAZ FILHO, A. C.; VITOR, P. C. G. Avaliação de diferentes hipsômetros na estimativa da altura total. Revista Verde de Agroecologia e Desenvolvimento Sustentável, Pombal, v.11, n.2, p. 01-05, 2016.
JESUS, C. M.; MIGUEL, E. P.; LEAL, F. A.; ENCINAS, J. I. Avaliação de diferentes hipsômetros para medição da altura total em um povoamento clonal de Eucalyptus urophylla $\mathrm{x}$ Eucalyptus grandis. Enciclopédia Biosfera, Goiânia, v. 8, n. 15, p. 291-299, 2012.

MACHADO, S. A.; FIGUEIREDO FILHO, A. Dendrometria. Curitiba: Edição dos autores, Embrapa Florestas. v.1, 2003. 309p.

OLIVEIRA, X. M.; OLIVEIRA, R. R.; RAMALHO, F. M. G.; CABACINHA, C. D.; ASSIS, A. L. Precisão e tempo de operação de alguns instrumentos para medir altura de árvores. Enciclopédia biosfera, Goiânia, v.10, n.18; p.2336-2344. 2014.

R Core Team. R: A Language and Environment for Statistical Computing. R Foundation for Statistical Computing. URL http://www.Rproject.org/, Viena. 2016.

SANQUETTA, C. R.; CORTE, A. P. D.; MOGNON, F.; RODRIGUES, A. L. Estimativa de carbono individual para Araucária angustifólia. Pesquisa Agropecuária Tropical, Goiânia, v.44, n.1, p.1-8, 2014.

SCOLFORO, J. R. S.; THIERSCH, C. R. Biometria Florestal: medição, volumetria e gravimetria. Universidade Federal de Lavras, Lavras, 2004. 285p.

SILVA, G. F.; CURTO, R. A.; SOARES, C. P. B.; PIASSI, L. C. Avaliação de métodos de medição de altura em florestas naturais. Revista Árvore, Viçosa, v.36, n.2, p.341-348, 2012a.

SILVA, G. F.; OLIVEIRA, O. M.; SOUZA, C. A. M.; SOARES, C. P. B.; LEMOS, R. Influência de diferentes fontes de erro sobre as medições de alturas de árvores. Cerne, Lavras, v.18, n.3, p. 397-415. 2012 b. 